\title{
Pratiques
}

Linguistique, littérature, didactique

\section{Faut-il réutiliser les concepts linguistiques du passé ? Un regard rétrospectif sur le prédicat}

Should the linguistic concepts of the past be reused? A retrospective look at the predicate

\section{Bernard Colombat}

\section{OpenEdition}

\section{Journals}

Édition électronique

URL : http://journals.openedition.org/pratiques/3791

DOI : 10.4000/pratiques.3791

ISSN : 2425-2042

Éditeur

Centre de recherche sur les médiations (CREM)

\section{Référence électronique}

Bernard Colombat, «Faut-il réutiliser les concepts linguistiques du passé ? Un regard rétrospectif sur le prédicat », Pratiques [En ligne], 175-176 | 2017, mis en ligne le 22 décembre 2017, consulté le 02 mai 2019. URL : http://journals.openedition.org/pratiques/3791 ; DOI : 10.4000/pratiques.3791

Ce document a été généré automatiquement le 2 mai 2019.

(c) Tous droits réservés 


\section{Faut-il réutiliser les concepts linguistiques du passé ? Un regard rétrospectif sur le prédicat}

Should the linguistic concepts of the past be reused? A retrospective look at the predicate

\section{Bernard Colombat}

1 Curieuse histoire que celle du prédicat ${ }^{1}$, qui fait couler tant d'encre dans les écoles depuis la rentrée d'octobre 2016. Une précaution préalable portera sur l'articulation de l'histoire des concepts et de l'histoire des termes. Nous partirons de cette remarque de G. Graffi (2008, p. 52, note 1) qui a consacré un article à l'histoire du sujet et du prédicat : « Pour des raisons de transparence terminologiques, je vais employer dans cet essai, au lieu d'"attribut", qui est plus commun en français, le terme de "prédicat", qui est quand même enregistré dans le Lexique de Marouzeau (1961) ».

2 La remarque soulève un problème intéressant: celui de l'adéquation des termes et des concepts, l'auteur préférant neutraliser la variété des termes pour concentrer son étude sur les concepts. Ce qui l'amène à préciser, lorsqu'il traite de Port-Royal : « Tant Caramuel que les Messieurs de Port-Royal font un usage généralisé non seulement des termes "sujet", "copule", "prédicat" (ou "attribut"), mais aussi de "proposition" [...]» (Graffi 2008, p. 63).

Or, si sujet, attribut et proposition sont bien présents dans la Grammaire générale et raisonnée, ni copule, ni prédicat ne se trouvent dans l'ouvrage, pas plus que prédicat n'est dans la Logique, ni d'ailleurs que dans aucune des autres grammaires françaises de la Renaissance et du XVII ${ }^{e}$ siècle $^{2}$. Et cela pose problème, car la neutralisation terminologique masque la distinction à faire entre une analyse à deux termes (sujet-prédicat) et une analyse à trois termes (sujet-copule-attribut). En effet Port-Royal définit ainsi la proposition: "Toute proposition enferme necessairement deux termes : l'un appellé sujet, qui est ce dont on affirme, comme terre; et l'autre appellé attribut, qui est ce qu'on affirme, comme ronde : et de plus la liaison entre ces deux termes, est. » (GGR, 1676, p. 29). 
On touche là du doigt une difficulté : celui de la copule, dont le statut spécifique - c'est un intermédiaire - « empoisonne » la vie des grammairiens depuis longtemps. Car si terre est le sujet, ronde l'attribut, est est ici simplement qualifié de liaison, alors qu'il est aussi qualifié dans la GGR $(1676$, p. 102, 120, 122, 129, 136) de "verbe substantif », selon une terminologie qui remonte à Priscien.

5 Remontons à Scaliger, qui occupe une place de choix dans l'horizon de rétrospection des Messieurs de Port-Royal. Toujours soucieux de trouver un fondement philosophique aux classes de mots, Scaliger oppose nom et verbe sur la base de l'expression de ce qui durable (apanage du nom) et ce qui est "fluent ", de ce qui s'écoule, exprimé par le verbe. Mais il rapporte également le verbe à l'expression de l'action et de la passion. C'est à ce sujet qu'il se heurte au verbe être. On peut trouver au verbe " un troisième mode » (outre l'expression de l'action et de la passion) : si je dis Caesar est clemens,

il apparaît qu'au lieu de signifier quelque chose, est est le signe de la liaison qui fait que la clémence est prédiquée en César. (ipsum est non uidetur aliquid significare, sed esse nota coniunctionis qua clementia in Caesare praedicatur, De causis linguae latinae, $1540,5,110$, p. 220).

6 Le voilà (sous la forme latine praedicatur), le terme recherché, dont - signalons-le au passage - il n'y a qu'une occurrence dans le De causis. Et Scaliger d'ajouter un peu plus bas :

Qu'il nous suffise, quant à nous, de diviser en deux l'ensemble du domaine des verbes : ceux qui signifient l'action et ceux qui signifient la passion, et d'y ramener tous les autres comme à des enseignes, ainsi que de réunir ces deux groupes en les ramenant à est. (ibid., p. 221)

7 Nous sommes alors tout près de la théorie de Port-Royal, et les Messieurs de Port-Royal auront beau jeu de critiquer Scaliger, mis «dans le même sac» que les autres prédécesseurs ${ }^{3}$, mais... d'en exploiter très certainement le texte pour proposer leur célèbre théorie de la proposition appuyée sur la copule comme élément de base, et la réduction de tout verbe, y compris le verbe substantif quand il marque l'existence, à la copule + un participe :

Quand je dis, Petrus vivit, Pierre vit: parce que le mot de vivit enferme seul l'affirmation, et de plus l'attribut d'estre vivant; et ainsi c'est la mesme chose de dire Pierre vit, que de dire, Pierre est vivant. (GGR, 1676, p. 96)

Nous voyons mesme que le verbe substantif sum, je suis est souvent adjectif, parce qu'au lieu de le prendre comme signifiant simplement l'affirmation, on y joint le plus general de tous les atributs, qui est l'estre; comme lors que je dis; je pense, donc je suis, je suis signifie là sum ens, je suis un estre, une chose : Existo signifie aussi sum existens, je suis, j'existe. (ibid., p. 120-121)

8 C'est la célèbre théorie du verbe ne signifiant que l'affirmation, restriction qui est poussée très loin, puisque «il n'y a que le verbe estre qu'on appelle substantif qui soit demeuré dans cette simplicité, et encore l'on peut dire qu'il n'y est proprement demeuré que dans la troisiéme personne du present, est, et en de certaines rencontres » (ibid., p. 96). En effet, si, par exemple, je passe à la première personne, je suis, j'introduis en effet un élément secondaire, la personne, de même que lorsque je change de temps ou que, comme supra, je fais exprimer l'existence au verbe être, etc.

Remontons dans le passé. C'était pourtant bien parti. Tout un chacun rappelle que le fondement de la division remonte à Aristote, qui pose un "substrat» ou un «sujet » ( hupokeimenon) et qu'on en dit quelque chose (kategorêma), avec cette particularité supplémentaire, comme le notent F. Ildefonse \& J. Lallot (2002, p.145), éditeurs des 
Catégories d'Aristote, que le vocabulaire des catégories est le même que celui de la prédication.

10 Mais dès l'origine, cette analyse fonctionnelle est entrée en concurrence avec une décomposition de l'énoncé sur la base de la distinction de deux catégories fondamentales de «mots »: dans le Cratyle, Platon nous dit que dans l'énoncé, il y a des noms (onomata) et des verbes (rhêmata), et dans le Sophiste, il en donne même une définition: les verbes désignent les actions, et les noms ceux qui font ces actions. La reconnaissance du nom et du verbe est donc assurée dès le IV $V^{e}$ siècle : une invention sans doute magnifique... mais dangereuse pour l'opposition fonctionnelle. Aristote identifie en outre l'arthron, qui n'est pas encore ce que nous appelons " article ", le sundesmos (la conjonction). Le système se perfectionne, les distinctions s'affinent. Dès lors, les deux perspectives, celle fondée sur une analyse des fonctions, et celle fondée sur la reconnaissance de mots, entrent en concurrence, alors qu'elles auraient pu être complémentaires.

11 En fait, dans le cas de langues flexionnelles, comme l'étaient le latin et le grec, on a pu "se passer » d'une analyse fonctionnelle. On avait des classes de mots, assez facilement identifiables, auxquelles on attribuait des «accidents» qui permettaient de les différencier et qui aidaient à établir leurs modes d'association. Le résultat final de cette association - qu'on l'appelle logos, oratio, phrase ou énoncé - n'est pas forcément l'objet principal de la réflexion. Prenons le cas de Priscien. Certes il nous dit - à la suite d'Apollonius Dyscole - que tout énoncé peut se réduire à un nom et un verbe (et non un sujet et un prédicat), que le nom doit précéder le verbe (GL 3 116-117). Mais il nous dit surtout que l'essentiel est d'associer correctement les différentes formes.

S'agissant donc de toutes les formes de ce genre, qui ont des significations variées, c'est la construction qui est absolument nécessaire pour les expliciter. Il en va de même aussi dans les noms, où l'on trouve des cas, des genres, des nombres différents ayant une forme identique, comme poetae, génitif et datif singulier (huius poetae / huic poetae), nominatif et vocatif pluriel (hi poetae / o poetae) [...].

On trouvera encore de multiples exemples du même type dans d'autres parties du discours, et l'on ne pourra analyser ces formes qu'en respectant la continuité logique de la construction (consequentia ordinationis), c'est-à-dire en distribuant convenablement les cas avec les cas, les genres avec les genres, les nombres avec les nombres, les personnes avec les personnes, les temps avec les temps. (Ars Prisciani, 17, 183-186 GL 3, 200.13-201.1, trad. A.g. 2010, p. 279)

L'histoire de la grammaire occidentale est ainsi devenue une histoire des parties du discours, voire une histoire de la combinatoire des catégories qui affectent ces parties du discours. Il y a sans doute plusieurs raisons à cela, mais une des plus importantes est sans doute celle-ci : on a pu assez facilement isoler des classes de mots sur des critères sinon définitifs, du moins assez stables pour perdurer, et on a pu se contenter de cette analyse4.

Revenons à la « disparition » du couple sujet-prédicat. L'intitulé d'un article de M. Baratin dans les Mélanges Collart (1978) est symptomatique à cet égard: "Sur l'absence de l'expression des notions de sujet et de prédicat dans la terminologie grammaticale antique ». L'expression est intéressante en soi : pourquoi ne trouve-t-on pas dans un texte ce qu'on s'attend à y trouver, ne serait-ce que parce que, avant, les notions ont existé ? La réponse est sans appel : les notions n'y sont pas, ou n'y sont plus, et à la place, il y a autre chose. Le couple subiectum vs praedicatum n'est pas passé dans la terminologie de la grammaire latine si l'on en croit le Lexicon de S. Schad (2007). L'opposition hupokeimenon/ kategorêma a certes été ainsi traduite par Boèce, traducteur d'Aristote, mais elle a été réservée, pour l'essentiel, à la logique. Parallèlement, les grammairiens ont été amenés à 
ré-élaborer un couple parallèle, celui de suppositum/appositum dont les origines sont obscures.

14 À vrai dire, de ces deux termes, c'est surtout l'histoire de suppositum qui a été étudiée (cf. Baratin, 1994 ; Graffi, 2008). Celle d'appositum est plus difficile à cerner. Dans le livre $17 \mathrm{de}$ l'Ars Prisciani, le verbe supponere n'apparait qu'une fois, alors que le participe passé suppositum est plus fréquent: dans tous les cas, il s'agit de faire référence à la réalité extralinguistique. Apponere est fréquent aussi, mais n'est en aucun cas opposé à supponere : il signifie «banalement » juxtaposer un élément à un autre. Il est cependant un passage du livre 18 où les deux verbes sont utilisés à quelques lignes de distance. Priscien nous dit que la plupart des verbes, «du fait qu'ils établissent transitivement une action à partir d'un nominatif vers des humains, se construisent avec l'accusatif, cependant que la personne qui exerce l'action et celle qui la subit sont l'une et l'autre supposées par le verbe " (utraque supponatur persona tam agentis quam patientis) : doceo te/doceor a te [je t'enseigne/je suis enseigné par toi]. Quand on tourne la phrase au passif, « celui qui subit l'action lorsque la signification est passive fait passer l'accusatif qui le concernait quand le verbe était employé à l'actif, en un nominatif associé au passif - nominatif qui s'entend dans le verbe lui-même (in ipso verbo intellegitur) [...], même s'il n'est pas présent à côté de lui (etiam si non apponatur) -, cependant qu'il fait passer à l'ablatif le nominatif correspondant à celui qui exerce l'action: erudio te, erudior a te [je t'instruis/je suis instruit par toi]. » (18, 139 GL 3 271.29-272.8 ; trad. A.g. 2017, p. 233).

Le texte utilise simplement ces deux verbes pour faire référence - négativement (non apponere) ou positivement (supponere) - au fait que la personne peut «ne pas être exprimée » ou «supposée ", et il ne dit rien de plus. Mais il est troublant de constater qu'il contient ces deux verbes, promis, avec leurs participes dérivés, à un bel avenir.

En tout cas, le suppositum est, au départ, très lié à la personne si l'on en croit A. de Libera \& I. Rosier (1992, p. 170) qui émettent l'hypothèse que "l'expression supponere verbo ("être le sujet du verbe") semble être une abréviation de supponere personam verbo [...], que l'on trouve ultérieurement sous la forme reddere personam verbo ou reddere suppositum verbo (Roger Bacon par exemple) ». Analysant le célèbre vers de Virgile, Vrbem quam statuo uestra est [La ville que je construis, c'est la vôtre], Robert de Paris (in Rosier, 1994, p. 114) nous dit: "Iste accusativus urbem construitur cum hoc verbo est et supponit ei personam ", litt. "Cet accusatif urbem est construit avec le verbe est et il met sous lui la personne ", alors même que ce serait au nominatif - attendu, Vrbs - d'assurer ce rôle.

À partir de là, la grammaire médiévale a établi une sorte de trilogie suppositum appositum obliquum qui n'est pas sans rappeler celle, plus récente, de "sujet verbe complément ", conférant au verbe le rôle d'« apposé ». De fait, c'est le suppôt qui concentre l'analyse. L'intérêt de l'analyse en termes de suppositum est de faire apparaitre des fonctions, indépendamment des caractéristiques des éléments qui peuvent occuper cette fonction. On en donnera un exemple par l'analyse des éléments - en apparence assez, voire très hétérogènes - qui peuvent occuper la fonction de suppositum dans le Catholicon de Giovanni Balbi (1286).

- Tout nom substantif est quelquefois suppôt par lui-même (supponit per se) : homo currit $(<1$ '/un> homme court).

- Ou un nom accompagné de sa 'terminaison', comme homo albus currit (<l'/un> homme blanc court).

- Pareillement est suppôt un nom adjectif placé comme substantif, comme album currit (<le/un> blanc court), c'est-à-dire alba res (<la/une< chose blanche).

- De même le participe comme legens proficio (litt. 'je-lisant je progresse'). 
- De même un verbe au mode infinitif placé comme nom est quelquefois suppôt par lui-même, comme legere est bonum (lire est bon) ; parfois accompagné d'un adjoint, comme [...] me ire ad ecclesiam est uerum (litt. moi aller à l'école = que j'aille à l'école est vrai), parce que ce tout me ire ad ecclesiam est sujet de ce verbe est à la place d'un nominatif.

- De même un verbe au subjonctif ou à l'indicatif, si un nom ou un adverbe indéfini est placé avant lui, comme qui currit scitur a me (litt. qui court est su de moi), ubi sit Sortes ignoratur a me (litt. où est Socrate est ignoré de moi).

- De même un énoncé constitué par le biais d'un verbe à l'indicatif ou au subjonctif (oratio sumpta per indicativum verbum vel subjunctivum) est suppôt par cette conjonction quod qui lui est préposée, comme quod tu legis est verum (le fait que tu lis est vrai) [...].

- De même est suppôt un adverbe placé en fonction de nom, comme diu est quod ego veni, c'est-à-dire diuturnum tempus (il y a longtemps que je suis venu). (Catholicon, éd. Mayence, 1460, f. 51 ${ }^{\mathrm{r} B}-51^{\mathrm{v}} \mathrm{A}$ ) le suppositum de ses réalisations morphologiques. À l'inverse, l'analyse de l'appositum n'a jamais été, à ma connaissance, aussi claire, peut-être parce que le verbe était presque toujours là, facilement identifiable, sans doute difficile à éliminer, et que d'autres analyses étaient possibles, par exemple en termes de transitivité de la personne passant, non d'un sujet à un objet, mais d'une personne à une autre (qui devait, selon les anciens, témoigner qu'elle subissait l'action de la première) par l'intermédiaire du verbe.

Si l'on fait un saut dans le temps, on retrouve au début de la Renaissance des traces de l'utilisation des termes suppositum/appositum. Mais ce qui domine alors, c'est une analyse morphosyntaxique, sur la base de l'opposition accord vs régime, la rection se situant non plus au niveau des fonctions, mais au niveau des parties du discours, voire à celui des catégories qui s'y appliquent. Le verbe est en effet l'élément pivot à partir duquel s'analysent les autres éléments. Donnons par exemple la définition du verbe chez Guarino de Vérone $\left(\mathrm{XV}^{\mathrm{e}} \mathrm{s}\right.$.) :

Remarque que le verbe actif acquisitif (verbum actiuum acquisitiuum) est celui qui veut avant lui le nominatif de la personne agissant et après lui l'accusatif de la personne subissant, et en outre un datif d'après la nature de l'acquisition, par ex. do panem pauperi « je donne du pain au pauvre».

La définition fait intervenir la notion de rection ou de dépendance (exprimée par le terme uelle) et intègre la rection du nominatif par le verbe; on remarquera l'utilisation des termes persona agens " personne agissant", persona patiens " personne subissant», qui sont en l'occurrence désémantisés («l'accusatif de la personne subissant », c'est panem dans l'exemple donné), de même que l'ordre fixe des constructibles (uelle ante se, post se). La présentation intègre toutes les constructions verbales et s'étend à la copule qualifiée de verbum neutrum similium copulatiuum (verbe neutre associant des éléments semblables).

1 Parallèlement s'est développée dans la tradition grammaticale une autre analyse $d u$ couple suppôt et appôt, compris non plus en termes de sujet vs prédicat, mais en termes de nom sujet vs nom placé après le verbe, comme le montre cette remarque de J. Despautère qui prouve qu'il n'y a pas unanimité sur la définition : dans les structures du type de celles qui viennent d'être décrites, «le nominatif est communément appelé suppôt, et le verbe appôt, mais il serait plus juste d'appeler appôt le cas du verbe que ce verbe régit après lui ${ }^{5}$ ».

2 La situation perdure durant les XVI et XVII ${ }^{e}$ siècles, comme le montrent les attestations nombreuses de l'opposition chez Sanctius, Scioppius et Vossius qui ne voient plus dans l'appôt que le nom en fonction d'objet (cf. Graffi 2008, p. 62-63).

Il est intéressant aussi de voir que les termes passent dans quelques-unes des premières grammaires françaises. Il est encore plus intéressant de voir que l'appost peut désigner : 

convenance [l'accord] du nom avec le verbe et en nombre et personne » :

Le nom et isi apele supos, le verbe apos. (GramerĘ, 1562, p. 90)

Le nom precedent deuant le verbe est icy appelle suppost, Le verbe appost /

Le nom prẻsẻdẻnt devant le vẻrbe ẻt isi apele supo, Le vẻrbe apo. (Grammaire, 1572, p. 153)

25

- soit le nom qui suit le verbe, ainsi chez L. Meigret qui - réorientant, voire inversant l'opposition - utilise surposé ou apposé pour le nom qui gouverne (régit) le verbe et sousposé pour celui qui est gouverné :

J'appelle le nom surposé ou apposé, celui qui gouverne le verbe et le sousposé ou souposé celui qui est gouverné : comme Pięrr' eyme Laorȩns, là où Pięrre est le surposé et Laorẹns le sousposé : ce que ne se doit pas entendre selon l'ordre des paroles, mais selon le sens : car celui qui gouverne est réputé es verbes actifs comme agent, et celui qui est gouverné comme patient: et au contraire es verbes passifs : car lors le surposé est le patient et le sousposé l'agent accompagné de de, du ou par ou dess : comme Pięrr' ȩt battu de Ian ou par Ian ou par brigans ou dȩ' brigans. ( Le trĘtté de la grammĘre françoĘze, 1980 [1550], p. 52)

L. Meigret prévoit également le cas du nom placé après la copule :

Il faut aussi entendre que le seul verbe substantif suys, ȩs, et veut toujours un sousposé nominatif: comme je suys Pięrre, tu ȩ' Pięrre, il ȩt Ian : mais, quand il signifie "appartenir ", il ne le peut souffrir : et faut lors que le sousposé soit gouverné par quelque préposition : comme je suys a vous: au regard de je suys de Líon : çet' imaj' ȩt de Pięrre et autres semblables façons de parler, elles suppléent le participe qui leur est requis : comme né, fętte : quasi voulant dire je suys né ou natif de Líon : çet' imaj' ȩt fẹtte de Pięrre. (ibid.)

Mais c'est bien la place que le terme désigne, non la fonction, le nom sousposé s'appliquant aussi bien à ce que nous appelons attribut - et alors il est au "nominatif ", c'est-à-dire qu'il se construit directement après la copule - qu'au syntagme prépositionnel (de + nom), et alors il désigne ce que les autres grammairiens $d u X V I^{e}$ siècle qualifient, moins prudemment que L. Meigret, d'« ablatif ».

Nos remarques sur la suite de l'histoire seront beaucoup plus succinctes. Littéralement, l'analyse en sujet - prédicat est inconnue des grammairiens français des XVII ${ }^{\mathrm{e}}$ et $\mathrm{XVIII}^{\mathrm{e}}$ siècles, puisqu'ils préfèrent parler d'une opposition en sujet et attribut : la place du verbe dans ou hors de cet attribut peut leur faire problème, Du Marsais disant que L'attribut contient essentiellement le verbe, parce que le verbe est dit du sujet, ce que N. Beauzée reformule ainsi: «Disons donc avec notre grammairien philosophe, que l'attribut commence toujours par le verbe » (Grammaire générale, 1767, t. 2, p. 8-10).

$\mathrm{Au} \mathrm{XX}^{\mathrm{e}}$ siècle, dans la tradition occidentale, c'est le terme prédicat qui s'impose définitivement dans ce sens - attribut étant désormais réservé à une fonction de l'adjectif -, et ce même si le concept qu'il désigne est parfois remis en cause, en même temps que l'opposition sujet vs prédicat fait l'objet de nombreuses remarques, que nous ne pouvons évoquer ici dans le détail. Nous renvoyons le lecteur aux sites CTLF et Frantext-CTLF ${ }^{6}$ qui permettent de nombreuses recherches combinées et qui montrent - on s'en doute, mais peut-être n'est-il pas utile de le rappeler - que la citation d'un terme, ou d'un couple de termes - ne signifie pas forcément adhésion à son utilisation.

Nous ne retiendrons ici que quelques citations particulièrement symptomatiques chez quelques auteurs. 
31 1. Du côté de ceux qui considèrent que la phrase de base est constituée de sujet prédicat :

32 - C. Bally (1965) fait un constant usage de l'opposition, et explique par ex. comment on est passé de Deus! Bonus! à Deus est bonus :

On peut supposer que, dans un état primitif que nous symbolisons, pour plus de commodité, par des mots latins, on a dit d'abord : Deus est! Bonus! « Il y a un Dieu! (Il est) bon ». D'autre part, on connaissait le dirème Deus bonus " Dieu (est) bon », qui s'est appliqué analogiquement sur ce système de coordonnées. Le verbe qui y figure est apparu comme un moyen commode d'exprimer à la fois la transposition de bonus en prédicat et la liaison, jusqu'alors implicite, entre le sujet et ce prédicat. Ainsi Deus est bonus n'a plus formé qu'une phrase liée, et le verbe a passé du premier membre dans le second, car tout ligament est attaché au prédicat ou au déterminant. (ibid., § 102, p. 72)

Toute énonciation comprend logiquement deux termes, la chose dont on parle et ce qu'on en dit; ce qu'on en dit est le propos ou prédicat (dans le sens large); le terme qui est l'occasion du propos est le thème ou sujet (dans le sens large). (ibid., § 154, p. 101)

On ne peut imaginer d'énoncé sans qu'on dise ou pense à propos de quoi il est fait. On retrouve ce genre de rapport dans tous les types de syntagmes : pas de sujet sans prédicat et vice versa; pas de copule sans un terme qu'elle rattache au déterminé (par exemple : pas de verbe transitif sans un complément d'objet ; inversement, pas de complément d'objet sans un verbe transitif, etc.). (ibid., § 155, p. 102)

- C.-A. Sechehaye (1926) considère comme centrale la phrase à sujet et prédicat :

Dans le développement syntaxique de la langue comme instrument de pensée et de communication, le type de la phrase-idée ne joue qu'un rôle secondaire, tandis que celui de la phrase-pensée, c'est-à-dire de la phrase à sujet et à prédicat, est devenu le principe générateur, l'organe central de tout le mécanisme grammatical.

Il est vrai que de modification en modification la phrase à sujet et à prédicat dont on vient de voir les divers types successifs a beaucoup changé de nature. Y a-t-il encore quelque chose de commun entre le dirème enfantin : Mama, obba! [Maman, lève-toi !] et une phrase exprimant une idée tout objective comme : Napoléon mourut à Sainte-Hélène? Nous avons essayé de le montrer ${ }^{7}$. (ibid., p. 30)

2. Du côté des linguistes hostiles à l'utilisation de l'opposition sujet vs prédicat :

Rappelons que ces termes de "subjectum » et de "prædicatum » proviennent de la terminologie de la logique aristotélique et qu'ils ont été introduits dans la grammaire par Boëce. Aussi longtemps qu'on maintiendra ces termes d'origine logique, il sera difficile d'affranchir des liens de la logique l'étude de ce moyen de communication intellectuelle qui a nom : le langage. (ibid., p. 5)

Mais on a aussi institué les catégories du sujet et du prédicat "psychologiques ». L'avantage de cette terminologie serait de pouvoir se passer de tout fait relatif à la forme. Cependant, ce système ne fait pas suffisamment droit à la nature pratique du langage. On ne peut tenir compte de celle-ci qu'en abandonnant toutes ces dénominations de : proposition (= jugement logique), sujet, prédicat, empruntées à la logique. A cette condition-là on a pleine liberté de traiter le langage, comme on doit véritablement le faire, c'est-à-dire comme le moyen par excellence de communication des hommes entre eux. (ibid., p. 6)

- alors que L. Tesnière (1965) critique également l'analyse logique traditionnelle :

2. - Se fondant sur des principes logiques, la grammaire traditionnelle s'efforce de retrouver dans la phrase l'opposition logique entre le sujet et le prédicat, le sujet étant ce dont on dit quelque chose, le prédicat ce qu'on en dit. 
3. - Ainsi dans la phrase Alfred parle lentement, le sujet serait Alfred et le prédicat parle lentement [...].

4. - Il ne faut voir dans cette conception qu'une survivance non encore éliminée, de l'époque, qui va d'Aristote à Port-Royal, où toute la grammaire était fondée sur la logique.

5. - En effet tous les arguments qui peuvent être invoqués contre la conception du nœud verbal et en faveur de l'opposition du sujet et du prédicat relèvent de la logique formelle a priori, qui n'a rien à voir en linguistique.

6. - Quant à l'observation strictement linguistique des faits de langue, les conclusions qu'elle permet a posteriori sont de tout autre nature. Dans aucune langue, aucun fait proprement linguistique n'invite à opposer le sujet au prédicat. ( ibid., p. 103-104)

3. Sur le fait que la phrase à sujet prédicat n'est pas la seule structure possible :

- A. Meillet (1958) estime qu'il faut s'entendre sur la définition du terme prédicat : Sous l'influence de la logique formelle qui, jusqu'au début du XIXe siècle, a dominé toutes les théories grammaticales, par suite aussi de l'habitude de fonder les théories linguistiques sur des formes de la langue écrite, on s'est longtemps imaginé que toute phrase comporte naturellement un «sujet » et un "prédicat». La phrase à terme unique apparaissait dès lors comme incomplète : dans une phrase composée du prédicat seul, il y aurait « ellipse » du sujet.

Pour qu'il y ait phrase, il faut et il suffit que quelque chose soit énoncé ; ce peut être un fait particulier ou une vérité générale que l'on indique, un sentiment qu'on exprime, un ordre qu'on formule. On peut convenir d'appeler « prédicat » ce qui est ainsi énoncé. Mais il faut retenir que la phrase ainsi définie diffère des «propositions » de la logique formelle, et, par suite, le terme de "prédicat » n'est pas ambigu. (ibid., p. 2)

- A. Martinet (1993) montre qu'il y a des prédicats tout seuls.

Quelque fréquent que soit le type d'organisation sujet-prédicat, on aurait tort de le croire universel. Il ne manque pas de langues où un énoncé parfaitement normal comporte un seul monème qu'on pourrait traduire par "pluie ", pour « il pleut ", par « renard», pour «voici un renard», etc., et ceci non seulement dans les cas marginaux que représentent les injonctions et les formes elliptiques de communication, mais aussi dans les messages proprement énonciatifs. (ibid., p. 126-127)

\section{Conclusion}

Pour qui veut établir la genèse des deux dichotomies, sujet/prédicat et suppôt/appôt, il est sans doute plus facile de discerner la première - l'opposition logique -, avec le sujet, «ce qu'on pose » au départ, et le prédicat, «tout ce qu'on dit du sujet ", même si c'est assez vaste et si ça reste assez vague. Pour l'opposition grammaticale, suppôt et appôt, c'est plus difficile : suite à une origine obscure, les deux termes ont gardé une certaine opacité. Le suppositum a été le référent extralinguistique avant de désigner la personne posée sous le verbe, puis le sujet grammatical. Si le terme est bien le résultat de l'abréviation de supponere personam verbo, « mettre la personne sous le verbe » ou « tenir lieu de personne pour le verbe ", cela révèle une filiation opaque, à mettre en lien avec la notion - toujours essentielle dans la grammaire ancienne - de personne, un terme dont le sens s'est dévoyé ou dilué (comme supra dans la formule « l'accusatif de la personne subissant » appliqué à «du pain» dans je donne du pain à un pauvre). Quant à l'appôt, il a donné lieu à des interprétations encore plus différentes : est-il tout ce qui n'est pas suppôt ? le verbe seul ? le nom "apposé » après le verbe? mais, alors, dans quelle fonction: attribut après le 
verbe être, ou complément du verbe transitif ? À ma connaissance, la délimitation de l' appositum ne s'est jamais stabilisée et la clarification ne semble jamais avoir eu lieu. tous soient convaincus de sa validité, cf. C. Svedelius, L. Tesnière) à l'opposition sujet/ prédicat. Fallait-il la substituer à la trilogie «sujet verbe complément " qui était sans doute mal construite (deux termes désignant des fonctions combinés à un terme désignant une classe de mots), et qui mettait de côté la dichotomie initiale : parler, c'est souvent (mais pas toujours) dire quelque chose à propos de quelque chose (les anciens auraient peut-être plutôt dit au départ " évoquer une personne à propos d'une autre ", tant l'énoncé était fondamentalement pour eux l'expression d'une action exercée par un être sur un autre être)? Fallait-il écarter dans une première étape de l'analyse ce « complément » qui était tout à la fois une belle ouverture vers la diversité et une boite de Pandore?

Poser sujet vs prédicat, ou encore suppôt vs appôt, oblige à décomposer les problèmes en passant par des opérations binaires: Pierre de la Ramée, qui fondait son analyse sur des dichotomies, n'aurait sans doute pas désavoué le principe.

\section{BIBLIOGRAPHIE}

ARNAULD, A. \& LANCELOT, C. (1676) [1660]. Grammaire générale et raisonnée. Paris : P. Le Petit.

BALLY, C. (1965) [1932]. Linguistique générale et linguistique française. Berne : Francke.

BARATIN, M. (1978). «Sur l'absence de l'expression des notions de sujet et de prédicat dans la terminologie grammaticale antique ». In : Collart, J. (éd.), Varron, grammaire antique et stylistique latine. Paris : Les Belles Lettres, p. 205-209.

BARATIN, M. (1994). « Sur les notions de sujet et de prédicat dans les textes latins ». Archives \& Documents de la SHESL, seconde série, 10, p. 49-79. En ligne : http://www.persee.fr/doc/ hel_0247-8897_1994_num_10_1_3393?q=Sujet et prédicat dans la grammaire antique et médiévale.

BEAUZÉE, N. (1767). Grammaire générale. 2 tomes. Paris : J. Barbou.

COLOMBAT, B., FOURNIER, J.-M. \& AYRES-BENNETT, W. (éds) (2011). Grand Corpus des grammaires françaises, des remarques et des traités sur la langue (XIV ${ }^{e}-X V I I{ }^{e}$ siècles). Paris : Classiques Garnier Numérique.

DESPAUTÈRE, J. (1537). Commentarii gramatici. Paris : R. Estienne.

GIOVANNI BALBI. (1460) [1286]. Summa quae uocatur Catholicon. Mayence, s.n. Farnborough : Gregg International Publishers, 1971.

GRAFFI, G. (2008). « Subiectum et praedicatum de l'antiquité classique à Port-Royal ». Cahiers de l'ILSL 25, p. 51-68. En ligne : http://crecleco.seriot.ch/colloques/06STRUCTPROP/Graffi.pdf. GRouPe ARS GRAmmATICA (M. Baratin et al.) (éds) (2010). Priscien, Grammaire, livre XVII, Syntaxe, 1, texte latin, traduction introduite et annotée. Paris : Vrin. 
GROUPE ARS GRAMmATICA (M. Baratin et al.) (éds) (2017). Priscien, Grammaire, livre XVIII, Syntaxe, 2, texte latin, traduction introduite et annotée. Paris : Vrin.

GUARINO DE VÉRONE [GUARINUS VERONENSIS]. (1497) [ca 1418]. Grammaticales Regulae. Venise : Jo. de Quarengiis.

ILDEFONSE, F. \& LALLOT, J. (éds) (2002). Aristote. Catégories, présentation, traduction et commentaires. Paris : Éd. Le Seuil.

LALlOT, J. (1994) «Sujet/prédicat chez Apollonius Dyscole », Archives \& Documents de la SHESL, seconde série, 10, p. 35-47. En ligne : http://www.persee.fr/doc/ hel_0247-8897_1994_num_10_1_3392.

LIBERA, A. de \& ROSIER, I. (1992). «Chapitre 2. La pensée linguistique médiévale ». In : Auroux, S. (dir.), Histoire des idées linguistiques, vol. 2, Le développement de la grammaire occidentale. Liège : Mardaga, p. 115-186.

MAILlaRD, M. (2008). « Le prédicat. Comment sortir de la Tour de Babel ? » Lidil 37, p. 23-44. En ligne : http://journals.openedition.org/lidil/2686.

MARTINET, A. (1993) [1960]. Éléments de linguistique générale. Paris : A. Colin.

MEIGRET, L. (1550). Le trĘtté de la grammĘre françoĘze. Lyon : C. Wechel. Éd. F. J. Hausmann, avec orthographe modernisée, 1980, Tübingen : G. Narr.

MEILLET, A. (1958) [1921]. Linguistique historique et linguistique générale. Paris : H. Champion. NOVAKOVA, I. \& GUENTCHÉVA, Z. (éds) (2008). « Syntaxe et sémantique des prédicats ». Lidil 37. PRISCIEN. (1855-1859) [ca 525_526]. Ars Prisciani = Institutionum grammaticarum libri XVIII, éd. M. Hertz, in H. Keil ed., Grammatici Latini, Leipzig : Teubner.

RABY, V. (éd.) (2017). «L'énoncé dans les traditions linguistiques : logos, vākya, kalām, oratio et les autres ». Langages 202.

RAMUS, P. [LA RAMÉE, P. DE] (1560). Gramere [FransoezE]. Paris : A. Wechel. RAMUS, P. [LA RAMÉE, P. DE] (1572). Grammaire [Francoyse]. Paris : A. Wechel. ROSIER, I. (1994). « L'introduction des notions de sujet et prédicat dans la grammaire médiévale », Archives \& Documents de la SHESL, seconde série, 10, p. 83-119. En ligne : http://www.persee.fr/ doc/hel_0247-8897_1994_num_10_1_3394.

SCALIGER, J.-C. (1540). De causis linguae Latinae libri tredecim. Lyon : S. Gryphe. SCHAD, S. (2007). Lexicon of Latin Grammatical Terminology. Pise : Fabrizio Serra Editore. SECHEHAYE, C.-A. (1908). Programme et méthodes de la linguistique théorique. Genève : A. Eggimann. SECHEHAYE, C.-A. (1926). Essai sur la structure logique de la phrase. Paris : H. Champion. SÉRIOT, P. \& SAMAIN, D. (éds) (2008). « La structure de la proposition : histoire d'un métalangage ». Cahiers de l'ILSL 25.

SVEDELIUS, C. (1897). L'analyse du langage appliquée à la langue française. Upsala : Almqvist \& Wiksell. TESNIÈRE, L. (1965) [1953]. Éléments de syntaxe structurale. Paris : C. Klincksieck. 


\section{NOTES}

1. La littérature sur le sujet est immense, surtout si on la connecte avec la problématique de l'énoncé. Pour quelques points de repère, voir en particulier P. Sériot \& D. Samain (2008), I. Novakova et Z. Guentchéva (2008), M. Maillard (2008) et le récent numéro de Langages consacré à l'histoire de l'énoncé (Raby, 2017). L'article de G.Graffi (2008) dans le premier volume mentionné présente une des analyses les plus précises du couple parallèle suppositum appositum.

2. D'après B. Colombat, J.-M. Fournier et W. Ayres-Bennett (2011).

3. «Iules Cesar Scaliger a creu trouver un grand mystere dans son livre des principes de la Langue Latine, en disant que la distinction des choses in permanentes et fluentes, en ce qui demeure et ce qui passe ; estoit la vraye origine de la distinction entre les Noms et les Verbes : les noms estant pour signifier ce qui demeure, et les verbes ce qui passe. | Mais il est aisé de voir que toutes ces définitions sont fausses, \& n'expliquent point la vraye nature du Verbe. » (1676, p. 98-99).

4. Lallot (1994, p. 47), s'interrogeant aussi sur la « perte » de ce « trésor » qu'est l'analyse en sujet vs prédicat, mentionne déjà la responsabilité des stoïciens : «En évacuant le sujet, les Stoïciens ont fait voler en éclats le couple fonctionnel hupokeimenon- katêgoroumenon; en introduisant le 'cas direct' (ptôsis orthê) à la place du sujet, n'ont-ils pas préparé le terrain glissant qui allait conduire le grammairien, féru de morphologie, à bâtir sa syntaxe sur une opposition cas directcas obliques?».

5. "Nominatiuus uulgo dicitur suppositum, uerbum autem appositum : sed appositum rectius dixeris casum verbi quem post se regit. » (Commentarii gramatici, 1537, p. 200).

6. Aux adresses suivantes : http://ctlf.ens-lyon.fr/default.htm et http://www.frantext.fr/ctlf/.

7. Cf. aussi Programme et méthodes de la linguistique théorique (Sechehaye, 1908, p. 139) : «De même que le seul mot papa peut indiquer toutes sortes de pensées dans lesquelles l'idée du père de l'enfant joue le rôle principal, le mot dodo représentera toutes sortes de pensées dont le sommeil est l'élément dominant. Rapprochez ces deux symboles-phrases dans une synthèse suivant l'ordre de succession qui correspond à celui des idées, et dites par exemple : papa dodo pour dire : papa dort et vous avez créé la première phrase proprement dite. Maintenant analysez cet ensemble, et vous trouverez dans le mot papa la substance, le sujet de la déclaration, et dans le mot dodo l'attribut ou le prédicat, suivant que l'idée correspondante est conçue sous l'aspect de la qualité ou de l'action. »

\section{RÉSUMÉS}

Cet article a pour objet d'étudier l'histoire de la notion de prédicat, et surtout son absence pendant de longs siècles dans la tradition grammaticale occidentale. En effet, alors qu'Aristote fournissait les éléments nécessaires à une opposition entre "ce qu'on pose dessous ", sujet ou substrat (hupokeimenon), et «ce qu'on en dit » (kategorêma), les grammairiens, à la différence des logiciens, ont longtemps privilégié une analyse des relations qu'entretiennent les classes de mots distinguées par leurs «accidents» ou propriétés spécifiques. Les grammairiens médiévaux ont néanmoins éprouvé le besoin de réintroduire l'opposition ancienne sous la forme suppositum (suppôt) vs appositum (appôt), mais la définition de ce dernier ne s'est pas stabilisée, puisque l' appôt a pu désigner soit le verbe soit le nom qui suit le verbe. Port-Royal a proposé une analyse de 
la proposition en sujet vs attribut joints par une « liaison » constitué par le « verbe substantif » (le verbe être) considéré comme la matrice de tous les verbes. $\mathrm{Au} \mathrm{XX} \mathrm{X}^{\mathrm{e}}$ siècle, ce sont des linguistes comme C. Bally ou C.-A. Sechehaye qui ont revendiqué que la phrase de base devait être analysée en sujet et prédicat.

The purpose of this article is to study the history of the notion of predicate, and especially its absence for many centuries from the Western grammatical tradition. While Aristotle provided the elements necessary for an opposition between "what one puts underneath", subject or substrate (hupokeimenon), and "what one says about it" (kategorema), grammarians, unlike the logicians, for a long time privileged an analysis of the relations between the word classes distinguished by their "accidents" or specific properties. Medieval grammarians nevertheless felt the need to reintroduce the old opposition between suppositum and appositum, but the definition of the latter did not become fixed, since the appositum could designate either the verb or the noun following the verb. Port-Royal proposed an analysis of the proposition into subject vs. attribute joined by a "liaison" (link) constituted by the verb to be ("substantive verb"), considered as the matrix of all verbs. In the 20th century, it was linguists such as C. Bally or C.-A. Sechehaye who claimed that the basic sentence should be analyzed into subject and predicate.

INDEX

Keywords : history of linguistics, parts of speech, predicate, subject, syntax

Mots-clés : histoire de la linguistique, parties du discours, prédicat, sujet, syntaxe

\section{AUTEUR}

\section{BERNARD COLOMBAT}

Université Paris Diderot/Université Sorbonne Nouvelle/Centre national de la recherche scientifique, HTL UMR 7597, F-75013, France 